\title{
When and how should we cluster and cross over: methodological and ethical issues (letter 1)
}

\author{
Jessica Spence, MD (1) - Emilie Belley-Côté, MD, FRCPC - Simon Oczkowski, MD, MSc, MHSC, FRCPC • \\ Eric Jacobsohn, MBChB, MPHE, FRCPC $\cdot$ Richard Whitlock, MD, PhD, FRCSC $\cdot$ P. J. Devereaux, MD, PhD, \\ FRCPC • Stuart Connolly, MD, FRCPC
}

Received: 16 June 2018/Revised: 16 July 2018/ Accepted: 16 July 2018/Published online: 17 October 2018

(C) Canadian Anesthesiologists' Society 2018

\section{To the Editor,}

The editorial by Goldstein et al. ${ }^{1}$ expresses concern regarding the methods and ethics of our BenzodiazepineFree Cardiac Anesthesia for Reduction in Postoperative Delirium (B-Free) trial, suggesting that waived consent cannot be justified when interventions are administered directly to individuals. In our view, however, international guidelines and current practice support the concept that trials evaluating treatments administered directly to individual patients can fulfill the requirements for a waiver of individual consent, provided that they have minimal risk.

There are many completed and ongoing cluster trials that test policies related to the use of pharmacologic and other interventions where waiver of consent has been accepted. Such trials include: conventional $v s$ incremental

This letter is accompanied by a reply. Please see Can J Anesth 2018; 65: this issue.

\section{J. Spence, MD ( $\square)$}

Departments of Anesthesia, Critical Care and Health Research Methods, Evidence, and Impact, Population Health Research Institute (PHRI), McMaster University, Hamilton, ON, Canada e-mail: jessicaspence13@gmail.com

\section{E. Belley-Côté, MD, FRCPC}

Departments of Medicine (Cardiology and Critical Care) and Health Research Methods, Evidence, and Impact, Population Health Research Institute (PHRI), McMaster University,

Hamilton, ON, Canada

S. Oczkowski, MD, MSc, MHSC, FRCPC

Department of Medicine (Critical Care), McMaster University, Hamilton, ON, Canada

E. Jacobsohn, MBChB, MPHE, FRCPC

Departments of Anesthesia and Internal Medicine, University of Manitoba, Winnipeg, MB, Canada

\section{R. Whitlock, MD, PhD, FRCSC}

Departments of Surgery (Cardiac Surgery) and Health Research Methods, Evidence, and Impact, Population Health Research Institute (PHRI), McMaster University, Hamilton, ON, Canada

\section{P. J. Devereaux, MD, PhD, FRCPC}

Departments of Medicine (Cardiology) and Health Research Methods, Evidence, and Impact, Population Health Research Institute (PHRI), McMaster University, Hamilton, ON, Canada

S. Connolly, MD, FRCPC

Department of Medicine (Cardiology), Population Health Research Institute (PHRI), McMaster University, Hamilton, ON, Canada 
antibiotic prophylaxis strategies on the incidence of pacemaker infection; ${ }^{\text {A }}$ saline compared with buffered crystalloid intravenous fluid on the incidence of acute kidney injury; ${ }^{\mathrm{B}}$ chlorhexidine-impregnated $v s$ nonantimicrobial wipes on healthcare-associated infection ${ }^{\mathrm{C}}$ and empiric antibiotic cycling on the prevalence of antibiotic resistant bacteria in intensive care unit patients. ${ }^{\mathrm{D}}$ Trials using individual randomization have also satisfied the requirements for waiver of consent, including a recent large trial examining the effect of fresh $v s$ older blood on mortality after transfusion. ${ }^{\mathrm{E}}$ Although the authors of the editorial take an extreme position that informed consent is always required for individual-level interventions, one of the authors (Taljaard) was an investigator in a recently published trial comparing high $v s$ low-dose influenza vaccination in individual residents of nursing homes, where consent to participate was obtained from the nursing home (i.e., gatekeeper consent), not the individual residents. ${ }^{\mathrm{F}}$

Various regulatory bodies overseeing and ensuring the ethical conduct of research, such as the Government of Canada Panel on Research Ethics and the United States Food and Drug Administration, have established requirements to justify a waiver or modification of individual patient consent. ${ }^{2,3}$ They incorporate the following general principles:

\footnotetext{
A U.S. National Library of Medicine. Prevention of Arrhythmia Device Infection Trial (PADIT). ClinicalTrials.gov identifier: NCT01628666. Registered June 27, 2012. Available from URL: https://clinicaltrials.gov/ct2/show/NCT01628666 (accessed July 2018).

B Australian New Zealand Clinical Trials Registry. Trial identifier: ACTRN12613001370796. 0.9\% Saline vs. Plasma-Lyte 148 for Intensive Care Fluid Therapy (the SPLIT study). Registered December 13, 2013. Available from URL: https://www.anzctr.org. $\mathrm{au} /$ Trial/Registration/TrialReview.aspx $? \mathrm{id}=365460$ (accessed July 2018).

C U.S. National Library of Medicine. ClinicalTrials.gov identifier NCT02033187. Daily Chlorhexidine Bathing and Infection Rates in Critically-ill Patients. Registered January 10, 2014. Available from URL: https://clinicaltrials.gov/ct2/show/NCT02033187 (accessed July 2018).

D U.S. National Library of Medicine. Effect of Antibiotic Rotation in the ICU on the Prevalence of Antibiotic Resistant Gram-negative Colonisation (SATURN). ClinicalTrials gov identifier NCT01293071. Registered February 10, 2011. Available from URL: https:// clinicaltrials.gov/ct2/show/NCT01293071 (accessed July 2018).

E ISRCTN Registry. Identifier ISRCTN08118744. Informing fresh versus standard issue red cell management. Registered February 16, 2012. Available from URL: http://www.isrctn.com/ ISRCTN08118744 (accessed July 2018).

F U.S. National Library of Medicine. ClinicalTrials.gov identifier NCT01815268. High-Dose Influenza Vaccine in Nursing Homes. Registered March 21, 2013. Available from URL: https:// clinicaltrials.gov/ct2/show/NCT01815268 (accessed July 2018).
}

(i) altered consent is required to answer the research question

(ii) research involves minimal risk

(iii) lack of a priori consent will not adversely affect participant welfare

(iv) information about the research being conducted is provided to participants when possible

(v) benefits of undertaking the research outweigh the risks of not obtaining a priori consent.

Goldstein et al. assert that the intervention evaluated within the context of the B-Free trial neither requires cluster randomization nor satisfies criteria for waiver of individual consent. In particular, they assert that altered consent is not required to answer the research question. We disagree, and perhaps they have misunderstood the question that the B-Free trial is addressing. Our trial is not asking what happens to individual patients randomized to receive one therapy or another. Rather, we are asking what happens to hospital delirium incidence when there is an institutional policy of one therapeutic strategy compared with another. This question can only be answered by randomizing at the institutional level, as in the cluster cross-over trial that we are conducting. Cluster randomized trials are the preferred design to address questions of institutional policy, whether applied directly or indirectly at the level of the individual. For the most part, anesthesia practice is based in institutions treating many patients with similar conditions. Patient care is ideally guided by institutional policies that incorporate best practice when this is well-defined. Many factors may impact effectiveness, beyond the efficacy of the policy itself. Specifically, issues around practitioner adherence to the policy (reflecting knowledge translation) or policy application at the level of the individual patient (reflecting population selection) are not taken into account in individual participant randomized trials but are captured by cluster trials.

B-Free evaluates two different cardiac anesthesiology policies related to the use of benzodiazepines (minimized $v s$ liberal intraoperative administration). These different approaches are both minimal risk and used commonly within Canada, ${ }^{4}$ though their relative effects are poorly understood. In our trial, patients receive a letter of notification in advance of their surgery that provides information about the trial. Patients are notified that anonymized hospital data will be collected and are given the option to withdraw their data. In a pilot study conducted in preparation for the main B-Free trial, ${ }^{5}$ which included 800 patients in two centres, no patient asked to have their data withdrawn. Given that we are testing the effects of institutional policy change, the intervention is of minimal risk, and we include patient 
notification as an element of trial procedures, we maintain that the B-Free trial is ethical. Once complete, the trial will generate important information that will guide institutional cardiac anesthesia policies and may reduce the incidence of postoperative delirium.

Financial disclosure and conflicts of interest None declared.

Editorial responsibility This submission was handled by Dr. Gregory L. Bryson, Deputy Editor-in-Chief, Canadian Journal of Anesthesia.

\section{References}

1. Goldstein CE, Giraudeau B, Weijer C, Taljaard M. When and how should we cluster and cross over: methodological and ethical issues. Can J Anesth 2018; 65: 760-5.

2. Canadian Institutes of Health Research; Natural Sciences and Engineering Research Council of Canada; Social Sciences and
Humanities Research Council of Canada. Tri-Council Policy Statement: Ethical Conduct for Research Involving Humans December 2014. Available from URL: http://www.pre.ethics.gc. ca/eng/policy-politique/initiatives/tcps2-eptc2/Default/ (accessed July 2018).

3. U.S. Department of Health and Human Services; Food and Drug Administration. IRB Waiver or Alteration of Informed Consent for Clinical Investigations Involving No More Than Minimal Risk to Human Subjects - July 2017 Available from URL: https://www. fda.gov/downloads/RegulatoryInformation/Guidances/UCM56694 8.pdf (accessed July 2018).

4. Spence J, Belley-Côté E, Devereaux PJ, et al. Benzodiazepine administration during adult cardiac surgery: a survey of current practice among Canadian anesthesiologists working in academic centres. Can J Anesth 2018; 65: 263-71.

5. U.S. National Library of Medicine. Benzodiazepine-free Anesthetic for Reduction of Delirium (B-Free). ClinicalTrials.gov identifier: NCT03053869. Registered February 15, 2017. Available from URL: https://ClinicalTrials.gov/ct2/ show/NCT03053869 (accessed July 2018) 